\title{
The ODD Old, Super-Metal-Rich Open Cluster, NGC 6791
}

\author{
Ann Merchant Boesgaard ${ }^{1,3}$, Michael G. Lum ${ }^{1,3}$ and \\ Constantine P. Deliyannis ${ }^{2,3}$ \\ ${ }^{1}$ Institute for Astronomy, University of Hawai'i at Manoa, \\ 2680 Woodlawn Drive, Honolulu, HI 96822 \\ ${ }^{2}$ Department of Astronomy, Indiana University \\ 727 East 3rd Street, Swain Hall West 319, Bloomington, IN 47405-7105 \\ ${ }^{3}$ Visiting Astronomer, W. M. Keck Observatory jointly operated by the California Institute of \\ Technology and the University of California.
}

\begin{abstract}
We report on the composition of turn-off stars in the intriguing open cluster, NGC 6791, which is old, but super-metal-rich using Keck/HIRES spectra. We find $[\mathrm{Fe} / \mathrm{H}]=+0.30$ $\pm 0.02[\mathrm{O} / \mathrm{Fe}]_{\mathrm{n}}-0.06 \pm 0.02,[\mathrm{Mg} / \mathrm{Fe}],[\mathrm{Si} / \mathrm{Fe}],[\mathrm{Ca} / \mathrm{Fe}]$, and $[\mathrm{Ti} / \mathrm{Fe}]$ near solar and the two Fe-peak elements, $\mathrm{Cr}$ and $\mathrm{Ni}$, are consistent with $\mathrm{Fe}$.
\end{abstract}

\section{Introduction}

NGC 6791 is a fascinating, unique cluster. For an open cluster it is very massive at $\sim 4000 M_{\odot}$ (King et al. $(2005)$ ). Its age is $\sim 8.3$ Gyr (Brogaard et al. $(2012)$ ). The metallicity is at least twice that of the sun (e.g., Boesgaard et al. (2009)). NGC 6791 has a heliocentric distance of $\sim 4 \mathrm{kpc}$ (King et al. (2005)). It has a Galactic latitude of $+11^{\circ}$ which makes it $1 \mathrm{kpc}$ above the Galactic plane. Most open clusters are near or in the Galactic plane. Bedin et al. (2006) found the absolute proper motion of the cluster. They determine orbital parameters and find that it has a boxy orbit with high eccentricity $(\epsilon$ $\sim 0.5$. Its perigalactic distance is about $3 \mathrm{kpc}$ and its apogalactic distance is about $10 \mathrm{kpc}$. The orbital period is $\sim 130 \mathrm{Myr}$ and it has crossed the Galactic plane several times. It has remained as an intact cluster due to its high mass and density. It may have originated in the inner regions of the Galaxy (Bedin et al. (2006)). Chemical evolution models are able to produce $[\mathrm{Fe} / \mathrm{H}] \leqslant+0.30$ for galactocentric distances of $<4-5 \mathrm{kpc}$ even at early ages. Following its formation in the inner part of the Milky Way, NGC 6791 may have been ejected into its current high eccentricity orbit by a massive feature, such as a bar (Jilkova et al. (2012)).

\section{Observations and Abundances}

Previous studies of $[\mathrm{Fe} / \mathrm{H}]$ in NGC 6791 have been mostly low and medium resolution, primarily of $\mathrm{K}$ and $\mathrm{M}$ giants. We chose to observe faint $(V \sim 17.4)$ main-sequence turn-off at high spectral resolution $(\sim 46,000)$ with the Keck $10 \mathrm{~m}$ telescope and its upgraded HIRES spectrograph. We used $B$ and $V$ values are from Stetson et al. (2003), the differential reddening corrections of Brogaard et al. (2012) and the calibrations of Casagrande et al. (2010) to determine temperatures. We also found temperatures spectroscopically from the agreement of Fe lines with a range of excitation potentials. The mean difference between $T_{\text {eff }} \mathrm{s}$ determined from the colors and those from excitation potentials is $-6 \mathrm{~K}$ $\pm 53 \mathrm{~K}$. The details can be found in Boesgaard et al. (2015). We used model atmospheres 

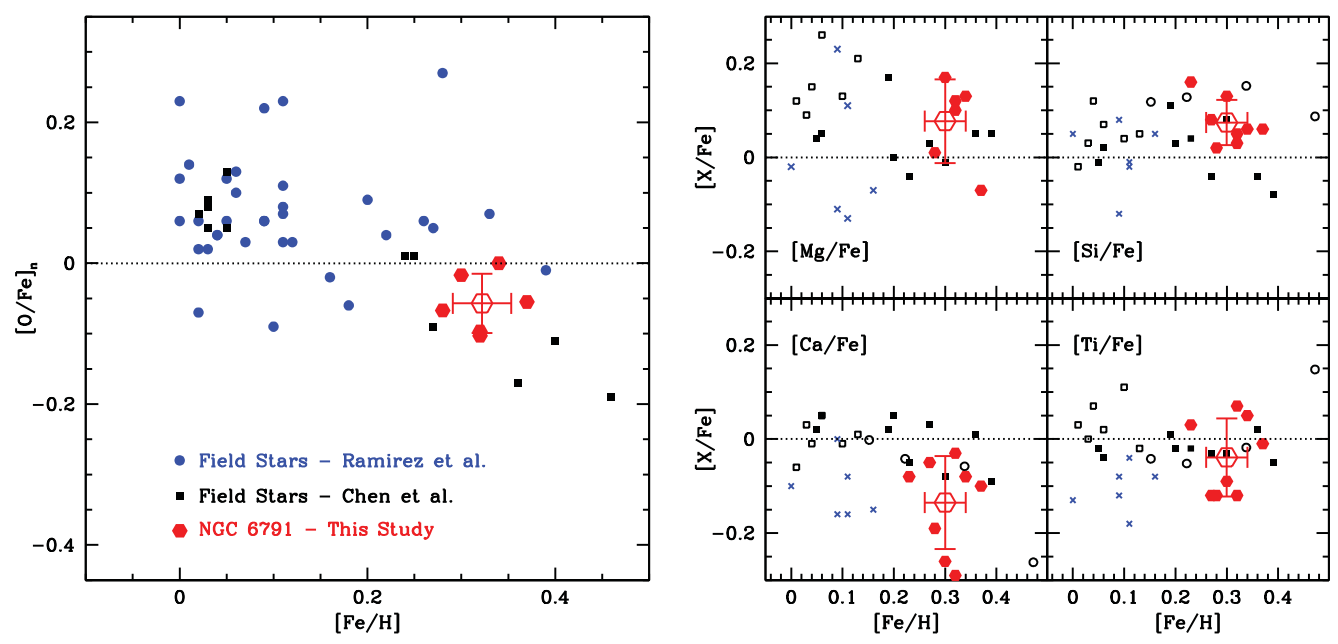

Figure 1. Left: Our results for $[\mathrm{O} / \mathrm{Fe}]$ as corrected for nLTE effects are the (red) hexagons and are compared with those from (Ramirez et al. (2013) and Chen et al. (2003). The stars selected from Ramirez are shown as filled (blue) circles and are metal-rich $([\mathrm{Fe} / \mathrm{H}]>0.00)$ and old (age >7.7 Gyr). The old (8-10 Gyr) metal-rich $([\mathrm{Fe} / \mathrm{H}]>0.01)$ field stars from Chen et al. (2003) are filled squares (black). The turn-off stars in NGC 6791 have values of $[\mathrm{O} / \mathrm{Fe}]_{\mathrm{n}}$ that are lower than solar $[\mathrm{O} / \mathrm{Fe}]_{\mathrm{n}}$. The trend of decreasing $[\mathrm{O} / \mathrm{Fe}]$ with increasing $[\mathrm{Fe} / \mathrm{H}]$ can be seen. Right: Abundances of the four alpha-elements relative to Fe shown as filled (red) hexagons. The cluster mean with error bars are shown as open hexagons. The comparison field stars are all old (>8 Gyr) and metal rich. The filled squares are from Chen et al. (2003), the open squares from Edvardsson et al. (1993), the open circles from Feltzing \& Gonzales (2001), the crosses (blue) are from Reddy et al. (2006) as selected by age and $[\mathrm{Fe} / \mathrm{H}]$ from (Ramirez et al. (2013).

of Kurucz (1993). We measured the equivalent widths for Fe I and Fe II lines which were unblended and unsaturated. The lines are unsaturated when $\log W / \lambda \leqslant-4.82$. We typically measured 37-46 Fe I lines and 5-8 Fe II lines. The values of $[\mathrm{Fe} \mathrm{I} / \mathrm{H}]$ and $[\mathrm{Fe}$ $\mathrm{II} / \mathrm{H}]$ agreed very well and the mean for the eight turn-off stars is $[\mathrm{Fe} / \mathrm{H}]=0.30 \pm 0.02$

The O I triplet lines at $7774 \AA$ are well-separated. In order to make a differential analysis with respect to the Sun, we used a 10 s exposure of the Moon that we had taken with Keck/HIRES in 2003 January 11 UT. The measured S/N in the region of the O I triplet is 350 . We measured the equivalent widths for the 3 lines in the lunar and stellar spectra and derived the LTE abundance for each line. Takeda (2003) has determined the nLTE corrections for each O I line for an array of temperatures, log $g$ values, and Fe abundances. We applied the appropriate corrections for each line by interpolating between Takeda's tables for our parameters. In Figure 1a (left) we show a comparison of our $[\mathrm{O} / \mathrm{Fe}]_{\mathrm{n}}$ versus $[\mathrm{Fe} / \mathrm{H}]$ with results for field stars. The main field star sample is from Ramirez et al. (2013), selected to have $[\mathrm{Fe} / \mathrm{H}]$ greater than 0.0 and ages older than 7.7 Gyr. Those 34 stars are from their total sample of 835 nearby FGK stars. Also shown in the figure are the $[\mathrm{O} / \mathrm{Fe}]$ and $[\mathrm{Fe} / \mathrm{H}]$ from the sample of old, metal-rich stars of Chen et al. (2003). These field stars are between 8-10 Gyr and have $[\mathrm{Fe} / \mathrm{H}]$ values $>0.00$. The values for $[\mathrm{O} / \mathrm{Fe}] \mathrm{n}$ in NGC 6791 turn-off stars fit the pattern of decreasing $[\mathrm{O} / \mathrm{Fe}]$ with increasing $[\mathrm{Fe} / \mathrm{H}]$. There is no sign of an intrinsic spread in $[\mathrm{O} / \mathrm{Fe}]_{\mathrm{n}}$ in our six turn-off stars, which is consistent with a single population. We determined abundances of the alpha-element from 2-3 lines of Mg I, 7-9 Si I lines, 7-9 Ca I lines and 6-8 Ti I lines. The cluster mean values are $[\mathrm{Mg} / \mathrm{Fe}]=+0.08 \pm 0.04,[\mathrm{Si} / \mathrm{Fe}]=+0.07 \pm 0.02,[\mathrm{Ca} / \mathrm{Fe}]$ $=-0.13 \pm 0.04$, and $[\mathrm{Ti} / \mathrm{Fe}]=-0.04 \pm 0.03$. Figure $1 \mathrm{~b}$ shows a plot of each of the four alpha-elements relative to $\mathrm{Fe}$ in each star as compared to the old and metal-rich field 
stars. The comparison stars are from Chen et al. (2003), Edvardsson et al. (1993), Feltzing \& Gonzales (2001), and Reddy et al. (2006). We were able to measure 5-6 unsaturated, unblended lines of Cr I and 11-16 unsaturated, unblended lines of Ni I. Both Fe-peak elements are similar to Fe in our turn-off stars. The comparison with old, metal-rich field stars hints that the NGC 6791 stars might possibly be slightly enriched in $\mathrm{Cr}$ and $\mathrm{Ni}$.

\section{References}

Bedin, L. R., Piotto, G., Carraro, G., King, I. R., \& Anderson, J. 2006, AE\&A, 460, L27 Boesgaard, A. M., Jensen, E. C., \& Deliyannis, C. P. 2009, ApJ, 633, 398

Boesgaard, A. M., Lum, M. G., \& Deliyannis, C. P. 2015, ApJ, 599, 202

Brogaard, K., VandenBerg, D. A., Bruntt, H. et al. 2012, AAP, 543, A106

Casagrande, L., Ramìrez, I., Melèndez, J., Bessell, M. \& Asplund, M. 2010 AAP, 512, 54

Chen, Y. Q., Zhao, G., Nissen, P. E., Bai, G., \& Qui, H. M. 2003, ApJ, 591, 925

Edvardsson, B., Andersen, J., Gustafsson, B. et al. 1993, A\&A A, 275, 101

Feltzing, S. \& Gonzales, G. 2001, A\&SA, 367, 253

Jilkova, L., Carraro, G., Jungwiert, B., \& Minchev, I. 2012, AAP, 541, 64

King, I. R., Bedin, L. R., Piotto, G., Cassisi, S., \& Anderson, J. 2005, AJ, 130, 626

Kurucz, R. L. 1993, CD-ROM 13 (Cambridge: Smithsonian Astrophys. Obs.)

Ramirez, I., Allende Prieto, C., \& Lambert, D. L. 2013, ApJ, 764, 78

Reddy, B., Lambert, D. L., \& Allende Prieto, C. 2006, MNRAS, 367, 1329

Stetson, P. B., Bruntt, H., \& Grundahl, F. 2003, PASP, 115, 413

Takeda, Y. 2003, A\&SA, 402, 343 